\title{
The experiences of young people with intellectual disability when transitioning to life after school: A systematic scoping review protocol
}

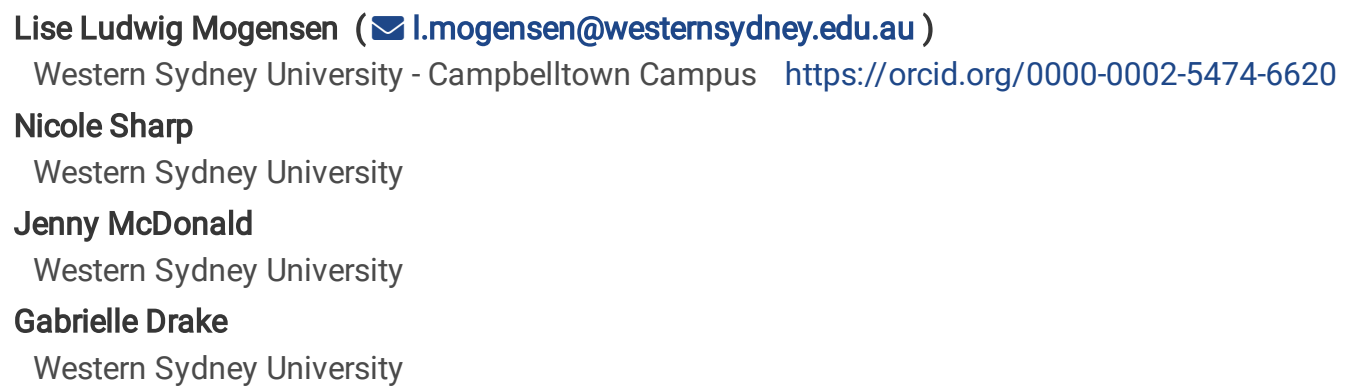

\section{Protocol}

Keywords: intellectual disability, transition, post-school, scoping review

Posted Date: October 28th, 2021

DOI: https://doi.org/10.21203/rs.3.rs-1017999/v1

License: (c) (7) This work is licensed under a Creative Commons Attribution 4.0 International License. Read Full License 


\begin{abstract}
Background: An increasing body of evidence suggests that transitioning from school to adult life can be challenging for young people with intellectual disability. Transition from secondary school includes preparation of students before leaving school and may continue several years into adult life. Transition challenges may include isolation, mental health problems, and reduced participation in employment and tertiary education. Some literature indicate links between poor post-school outcomes, and ineffective collaboration between school systems, disability services, and employers. Yet, consolidated evidence to understand how young people themselves experience transition from school is needed. The aim of this scoping review is to draw together current literature reporting what is important to young people with intellectual disability about transitioning to life after school.
\end{abstract}

Methods: Using the Joanna Briggs scoping review methodology, a systematic strategy will identify peer-reviewed qualitative research and grey literature reporting the voices of young people with intellectual disability to better understand personal experiences of transition to life after school. The review will explore scope and foci of topics, identify gaps in current evidence, and highlight approaches and methods used by investigators to include young peoples' own views.

Database searches for relevant qualitative research will include ERIC via Proquest, Ovid MEDLINE, selected EbscoHost databases, and Web of Science. Non-indexed and grey literature such as policies and reports will be searched via TROVE, ProQuest Dissertations \& Theses, and Google (with URL control and by crawling key agency websites). Studies will be selected using a three-step process: i) managing search results and removing duplicates, ii) title and abstract screening by two independent reviewers, and iii) full text review of included articles against inclusion criteria by two independent reviewers. Data will be tabulated, and presented in a narrative summary, aligning with the objectives and scope of this review.

Discussion: Review findings will inform policy makers, educators and disability support organisations of transition experiences of young people with intellectual disability. Their identification of transition challenges and enablers will inform the design and implementation of participatory transition processes, ensuring that future guidelines reflect the views of those they intend to support.

Registration with OSF DOI: 10.17605/OSF.IO/YHCDG

\title{
Background
}

\section{Transition from school - challenges and barriers}

Transitioning from school to post-school life and activities can be a challenging and stressful period, where young people seek to develop their own identity and identify opportunities for meaningful occupation, such as further study or employment (1). An increasing body of evidence indicates that young people with intellectual disability face additional barriers (2), including poor transition planning, and limited work experience opportunities(3). Despite the fact that transition policies and guidelines exist in several countries (4-6), these are not necessarily mandated, and may be difficult for young people and their cares to navigate $(2,3)$. Some studies have established links between poor post-school outcomes and ineffective collaboration between school systems and adult disability services as well as the paid and unpaid workforce (7). Other studies indicate young people with intellectual disability experience significant difficulty in negotiating the transition from a structured and supported school environment to contexts with 'wide variation in adoption of adult roles related to employment, independent living, friendships, and day activities' (8, p. 149). For many, the transition experience includes isolation, reduced participation in employment and tertiary education, and mental health difficulties (9). However, consolidated evidence on transition from school from the views and experiences of young people with intellectual disability themselves is wanting.

Understanding what is important to them is important in terms of ensuring policies and practices are designed to adequately respond to their needs.

\section{Views of parents on transition challenges}

Much of the existing evidence on young people with intellectual disability and the transition from school prioritises the perspectives of parents $(8,10)$. The important role of parents, especially mothers, in the transition process is well established $(8)$, and often outcomes are better for young people where their parents are actively involved in transition processes. Further evidence suggest that the post-school transition period can be highly stressful for families and carers of young people with intellectual disability (11). For example, Leonard et al (11) report parental concern about the ability of their adult child 'to adapt and change to life in adulthood' and worry about their child's long-term future. These concerns are based on parents reporting 'difficulty navigating services and programs', challenges for young 
people around 'building connectedness', along with family experiences of strain on wellbeing and finances (p. 1377). These findings are important and complex and suggest that challenges and enablers during transitions are highly context dependent.

\section{People with intellectual disability and participation in research}

People with intellectual disability are often marginalised within research, both as researchers and participants (12), despite evidence suggesting that many people with disability want to participate in research that is important to their lives (13). Grounds for exclusion include a history of exploitation of people with intellectual disability in research (14), beliefs that people with intellectual disability lack insight into their lives and needs, and so are less capable than others of contributing knowledge to research. The notion of vulnerability of certain populations, including children and people with disability, also means that gate keeping is common when it comes to participation in research and in decision-making processes. For example, the role of institutional ethical review boards on protecting people from harm can have the effect of excluding those who may be seen as unable to provide informed consent (15). Including children and young people with disability in research can be difficult, time consuming, and resource intensive for researchers (12). Parents, carers or organizational gatekeepers may also limit, delay or refuse access to potential participants. Including people with intellectual disability in research and understanding their views is critical to creating policies and practices that are responsive and accessible for those that they are designed to support (14). During the past 20 years, there has been a gradual paradigm shift toward more inclusive and participatory approaches to researching with people with intellectual disability, and greater recognition of the risks of paternalistic treatment (16). There is now an expectation in critical disability studies, that people with disability have agency and choice to lead, co-design, and/or participate in research about their lives (17). An initial search suggests that a developing body of literature exists in which young people's views and experiences on transition from school have been captured, but this has not been drawn together in a review to form a consolidated understanding of barriers and enablers to transitioning to meaningful life and occupations after school.

\section{Scoping review}

The aim of this scoping review is to identify, examine and synthesise the available evidence on the experiences of young people with intellectual disability transitioning from school to adult life from their own perspectives. The intent is to map out the existing literature on transitions from school from the past 20 years that reports the views and experiences of young people with intellectual disability. Further, the review aims to explore transition indicators and challenges that can better inform the development of future research, policies and transition guidelines framed by the voices of young people themselves, and to identify and analyse any knowledge gaps. It is the intention that this scoping review will inform the authors' research program, which explores from the views of young people with intellectual disability and other key stakeholders, the processes of transition from high school to adult life in Australia since the introduction of the publicly funded National Disability Insurance Scheme (NDIS) in 2013.

The NDIS is a consumer focused model funded through taxes and health care levies to provide reasonable and necessary supports for Australians with permanent and significant disability (18). The model takes a lifetime view of participant needs, and seeks early investment and intervention for people in order to maximise independence and social and economic participation and reduce long-term support requirements. The premise for the NDIS viability is the estimated efficiency gains and cost savings in the disability support system and savings to other government services.

Enabling 'people with disability to exercise choice and control in the pursuit of their goals and the planning and delivery of their supports' are the fundamental principles of the NDIS (18), an approach that represents a break from previous welfare approaches (19). The aim of the scheme is to empower people with disability, 'to use funds given to them to purchase services that reflect their lifestyles, goals and aspirations' $(19, \mathrm{np})$. This premise reflects the first principle of the United Nations Convention on the Rights of Persons with a Disability (UNCRPD), to which Australia became a signatory in 2008. The NDIS model is based on 'models of consumerism where those who consume services also have choice, participation, and rights and redress' (19). Thus, "choice," including choice during education and times of transition, is intended to be a central feature of the lives of people with disability.

For the purpose of this scoping review, transition is understood to include the time before young people leave secondary school, where the focus is on preparing for life after school, as well as the time after school where young people may be trialling options and making decisions about further education, work (paid and unpaid), lifestyle and relationships. We aim to include studies that explore all of these phases of transition to explore the experiences young people with intellectual disability as they move from high school to adult life.

In preparing this protocol, a preliminary literature search was conducted using ERIC and Ovid MEDLINE, using the terms "transition" and "intellectual disability." The pilot search was designed to broadly explore the types of evidence available, identify relevant key words and index terms and assess whether the research question had been previously addressed. While reviews exist on particular aspects or 
challenges of transition such as inter-agency collaboration (20), no current scoping or qualitative systematic reviews regarding the firsthand perspectives of young people with intellectual disability about their transition to life after school experiences were identified.

\section{Objective}

The objective of this scoping review is to identify and map published primary qualitative research as well as non-indexed and grey literature including policies, reports and guidelines that include the voices of young people with intellectual disability on their views and experiences of the transition from school to adult life. The protocol for the scoping review has been developed according to the Preferred Reporting Items for Systematic Reviews and Meta-Analyses for Protocols (PRISMA-P) statement using the checklist as attached in additional file 1 (21).

\section{Methods}

\section{Study design}

We chose a scoping review methodology for a number of reasons: Firstly, a scoping review is apposite to explore and map out the range of existing literature on a topic $(22,23)$ as in our case, reporting the voices of young people with intellectual disability on their experiences of transitioning from high school to adult life. In this respect, a review will report on the types of existing evidence in a topic area to help guide practice in a particular field. Secondly, a scoping review methodology is suitable for exploring broader questions on topics for which heterogeneity in methodology is expected (22). This allows for inclusion and review of academic publications as well as grey literature. Thirdly, a scoping review is useful in identifying gaps in existing evidence, clarifying key concepts, and thereby providing recommendations for future research and practice.

This scoping review will be conducted in accordance with the Joanna Briggs Institute (JBI) methodology for scoping reviews of qualitative evidence using Arksey \& O'Malley's (22) six step framework outlined in Table 1 with overview of the recommendations developed by Levac et al (24) and expansions by Peters et al (23). Additional file 2 provides a more detailed overview of this framework with descriptions of for each of these steps by Arksey \& O'Malley's (22) and recommendations by Levac et al (24) which are those utilised to develop the protocol.

A pre-registration for this review protocol has been submitted to the Open Science Framework (OSF) (DOI: 10.17605/OSF.IO/YHCDG).

Table 1

Scoping review framework

\section{Arksey and O'Malley framework (22p. 22-23) Enhancements proposed by Levac et al. (24p. 4-8)}

1. Identifying the research question

2. Identifying relevant studies

3. Study selection

4. Charting the data

5. Collating, summarizing and reporting the results

6. Consultation (optional)
Clarifying and linking the purpose and research question

Balancing feasibility with breadth and comprehensiveness of the scoping process

Using an iterative team approach to selecting studies and extracting data Incorporating a numerical summary and qualitative thematic analysis Identifying the implications of the study findings for policy, practice or research Adopting consultation as a required component of scoping study methodology

\section{The Scoping Review Framework}

\section{\#1 identifying the review questions}

The research questions for this review are relatively broad and were determined using the JBI Population, Concept, Context (PCC) framework to ensure that these align with inclusion criteria, data extraction and analysis. The research questions guiding this review are:

1. What literature exist that reports the lived experiences of young people with intellectual disability in the transition to life after school?

2. What transition indicators and challenges can be identified in this literature? 
3. What methods are used to hear the voices and experiences of young people with intellectual disability?

\section{\#2 Identifying relevant studies}

The population, concept and context (PCC) framework outlined in this section directed the research question and the development of the search strategy for this study.

\section{Population}

The population in this review will be young people with intellectual disability aged 15-30 years to capture a broad transition period. This age range reflects the varied definitions of what constitutes a young person. Increasing evidence also indicates that the transition to adulthood often extends well into the 20 s or even later $(1,25)$, particularly for people with intellectual disability $(26)$.

There is no universally accepted definition of intellectual disability, and language use changes over time and in different contexts. Therefore, for the purposes of this scoping review a broad approach to searching will be taken, utilising a range of current and older terms including disability or disabled or handicap; intellectual, learning, and developmental; cognitive impairment; and mental retardation to include US literature prior to 2013. The term 'mental retardation' is now considered derogatory in most English speaking countries, and was officially removed from the DSM-5 and ICD-11 in 2007, but was retained in some publications for several years. The use of the term also persisted in US federal policies until 2010, when President Obama signed the legislation required to replace the term with intellectual disability (27). The final rule was adopted in January 2013 (28).

\section{Concept}

The concept or phenomenon of interest to be explored in this scoping review is the experiences of young people with intellectual disability transitioning to life after school. This phenomenon incorporates three key phases for which varying terminology is used within the literature before, during and after transitioning. In the context of this scoping review, the search strategy will include terms that relate to:

1. planning for the end of secondary education, which should take place several years in advance,

2. the time of leaving high school and moving and settling into what comes next, for example,

3. living independently, community participation, education and training, and/or employment.

Research and grey literature about any post-school setting will be considered, including on post-secondary education, disability services, and the wider community. However, settings used as a main search string, may narrow the results significantly, so it is likely the settings will be considered in the extraction phase to help organise the review and analysis.

\section{Context}

Literature reporting young people's own experiences and views on the transition to adulthood will be prioritised to better understand what they consider important about their transition from school. Young people have a moral and ethical right to be involved in decisions that impact their lives, in line with the UN Convention of Rights of the Child (UNCRC) (26) and UN Convention of Rights of Persons with Disability (UNCRPD) (27). The searches will be for qualitative research involving young people with intellectual disability as participants, and grey literature that focuses on what is important to young people. Qualitative study designs including, but not limited to, phenomenological, grounded theory, ethnographic, action research and narrative inquiry will be considered. Papers that include a range of perspectives will be included so long as the views or experiences of young people themselves can be clearly distinguished in the findings. Where voices of parents, educators, service providers have been included, the reviewers will prioritise the perspectives of young people.

\section{\#3 Study selection Search Strategy}

To find both published studies and grey literature, a three step search strategy will be conducted in line with published scoping review guidelines (23). The first step will be a limited search of EBSCOhost and ERIC Databases, followed by an analysis of index terms and text words contained in the title and abstract of retrieved papers. This step will inform the development of a search strategy that will be tailored for each information source. A second search utilising all identified keywords and index terms will then be conducted across all included databases and repositories. Finally, the reference list of selected sources will be reviewed for additional sources.

The search strategy has been developed according to the Population, Concept, and Context as previously described, and in consultation with the University librarian. Synonyms and terms similar to named PCC concepts will be searched in strings with Boolean operator 'OR'.

Page 5/10 
Expanders will be applied to include equivalent subjects. PCC search strings will be combined with Boolean operator 'AND" in order to focus results to those applicable to our review. Truncation $\left(^{*}\right)$ will be used to find singular and plural forms of words and variant endings. Wildcard symbols (e.g. \$,?,\# depending on database) will be used to include various spellings of words if relevant. A preliminary search in CINAHL via EBSCOhost identified that it might be useful to use proximity operators to search phrases and key terms that are close in the text but not necessarily in the same order of appearance as the search phrase. The preliminary sample search strategy trialled in CINAHL is included in additional file 4.

The search for relevant qualitative published research will be conducted in selected EBSCOhost databases including PsychInfo, CINAHL Plus with Full Text, Education Research Complete; Psychology and Behavioral Sciences Collection; SocINDEX with Full Text, ERIC via Proquest, Ovid MEDLINE via Wolters Kluwer, and Web of Science via Clarivate. Non-indexed and grey literature such as policies, theses and reports will be searched via TROVE, ProQuest Dissertations \& Theses, and Google (with URL control or by crawling key agency sites for relevant papers and reports).

\section{Inclusion criteria}

This scoping review will include qualitative studies that explore young people's (aged 15-30 years) perspectives within published peer reviewed articles. Cultural, social, ethnic, and gender factors will be considered in the review of individual sources where these are clearly articulated. This review will include international literature, irrespective of country of origin, but limited to literature written in or translated to English, which is in line with the language proficiency of the reviewers. To ensure coverage of knowledge the review will also include grey literature including policies, guidelines, reports from advocacy groups, unpublished theses and relevant inquiries as long as the voices of young people with intellectual disability can be clearly distinguished.

The review will include sources published in the past 20 years since 2001. In this period, there has been increasing emphasis on the importance of hearing the voices of people with disability and facilitating their participation in research, labels and diagnoses have changed, and the UN Convention on the Rights of Persons with Disability (UNCRPD)(29) has been launched, ratified, and implemented to some extent in most countries.

All identified records will be uploaded into EndNote X9 (Clarivate Analytics, PA, USA) and Microsoft Excel, and duplicates removed. The titles and abstracts will be screened by two reviewers independently for assessment against the inclusion criteria for the review. Full text articles will be retrieved for all studies that meet the inclusion criteria of the review as well as those for which there are doubts. The reviewers will independently assess the full text in detail against the inclusion criteria. Full text papers that do not meet the specified criteria will be excluded, and reasons for exclusion will be recorded and reported in the final scoping review report. Any disagreements that arise between the reviewers will be resolved through discussion until consensus is reached, in conjunction with a third reviewer.

\section{\#4 Charting the data}

\section{Data extraction}

Data will be extracted from the included sources by two independent reviewers into a data charting template developed for this study. The data extraction tool will be adapted from Joanna Briggs Institute (23) and will include the key information listed in Table 2 along with variables to answer the research questions (amended points $5,6,7$ ). To pilot the extraction instrument, two reviewers will independently extract data from 5-10 included sources, and then discuss to determine whether the approach to extraction is appropriate and consistent. The tool will be revised if deemed necessary upon initial review of articles, and changes will be detailed in the full scoping review report. Any required data missing from or unclear in selected sources will be sought from authors where possible. 
Table 2

Proposed Data extraction tool categories

\begin{tabular}{|c|c|}
\hline 1 & Author(s) \\
\hline 2 & Journal and title of article \\
\hline 3 & Year of publication \\
\hline 4 & Origin/country of origin (where the source was published or conducted) \\
\hline 5 & Aims/purpose, key concepts: intellectual disability; transition; and participation \\
\hline 6 & $\begin{array}{l}\text { Participants characteristics and sample size within the source of evidence; young people with intellectual disability (or } \\
\text { synonym), age } 15-30 \text { years }\end{array}$ \\
\hline 7 & Methodology and methods of enabling young people 's participation in the research \\
\hline 8 & Contextual intersectional factors including setting, cultural, social, ethnic and gender if reported \\
\hline 9 & Data analysis - transition participation, indicators and challenges \\
\hline 10 & Authors conclusion \\
\hline 11 & Reviewer comments \\
\hline
\end{tabular}

\section{\#5 Collating, summarizing, and reporting the results}

The scoping review will be reported in line with the preferred reporting items for Systematic reviews and Meta-Analyses extension for Scoping Reviews (PRISMA-ScR) using the checklist in additional file 5. The numbers of sources of evidence screened will be presented in a flow diagram as illustrated in additional file 6 along with an explanation for the way decisions were made for inclusion or exclusion from the review at each stage. Each individual source will be listed with the relevant extracted data presented in a summary table in a manner that aligns with the questions and objective of this scoping review. A narrative review of the breadth and type of literature will accompany the tabulated results to describe how the results relate to the review objective and questions. The results will include qualitative content analysis of reported findings, and an outline of the identified gaps in the literature. The data for the qualitative analysis will be managed in NVIVO.

A conventional quality appraisal of included studies will not be conducted as this is not required for a scoping review. However, a summary and evaluation of methods used to ensure the inclusion of young people's voices in each source will be detailed. This will include, how young people with intellectual disability were recruited and informed about the research? How research participation was made accessible to people with intellectual disability? How the voices of young people with intellectual disability were clearly heard, or distinguished among other voices, or if appropriate whether their voices were absent or muzzled in the presentation? Harry Shier's model 'Pathways to Participation' (30) may be useful for this purpose. Although the model was developed for the purpose of evaluating the level of children's participation in decision-making, the five levels of participation (see Table 3) may be suitable to evaluate the participation of young people with intellectual disability. The model responds to the UNCRC (31) article 12.1 stating that 'State parties shall assure to the child who is capable of forming his or her own views the right to express those views freely in all matters affecting the child, the views of the child being given due weight...' This reflects some of the fundamental principles of the UNCRPD (29) including the rights of persons with disability to be directly involved and heard on issues that affect their lives. The suitability of Shier's model for appraising the methods and approaches to hearing and taking account of the voices of young people with disability, will be determined collaboratively by reviewers in the early stages of data extraction and review.

Table 3

Shier's Pathways to Participation

\begin{tabular}{|ll|}
\hline \multicolumn{2}{|l|}{ Five levels of participation: } \\
\hline 1 & Children are listened to \\
\hline 2 & Children are supported in expressing their views \\
\hline 3 & Children's views are taken into account \\
\hline 4 & Children are involved in decision-making processes \\
\hline 5 & Children share power and responsibility in decision making \\
\hline
\end{tabular}

Page $7 / 10$ 


\section{\#6 Consultation}

Arksey and O'Malley's original methodological framework for conducting scoping reviews highlighted consultation as an optional sixth stage of the review process (22). Levac and colleagues in 2010 recommended enhancements to the framework which included consultation as a required component of scoping reviews, designed to facilitate knowledge translation (24). This is consistent with the growing emphasis within broader health and disability research of the importance of reducing the knowledge-practice gap $(32,33)$. During this scoping review, and the team's broader program of research, regular consultation will be undertaken with an advisory group of young people with intellectual disability.

\section{Discussion}

Our preliminary literature search identified that there is an emerging body of literature exploring the experiences of young people with intellectual disability and their transition from high school to adult life, and that this time presents significant challenges to individuals and their families. It highlighted that a review to map and better understand this body of literature is warranted. A scoping review was selected as suitable in order to map out the extant qualitative academic and grey literature on transition from high school that include the voices and experiences of young people with intellectual disability. The intention is that this scoping review will inform research to explore from the views of young people with intellectual disability and other key stakeholders, the experiences of transition from high school to adult life in Australia, especially since the introduction of the publicly funded National Disability Insurance Scheme (NDIS) in 2013. The findings from this review will be published, and presented in conferences and public fora nationally and internationally. It is expected that the findings from this review will generate evidence on the challenges and enablers to meaningful transition planning and outcomes for young people with intellectual disability. The review will extend the current knowledge on transition planning, approaches and evaluation, and inform further policy development and guidelines for best transition practice in secondary education, employment, and the disability sectors.

\section{Declarations}

Ethics approval and consent to participate: not applicable

Consent for publication: not applicable

Availability of data and materials: not applicable

Competing interests: "The authors declare that they have no competing interests"

Funding: This review per se is not funded, but is part of a larger project on the young people with intellectual disability and the transition from secondary school to adult life funded by a grant from the Endeavour Foundation Disability Research Fund. The Endeavour Foundation Disability Research Fund aims to benefit Australians with a disability, especially people with an intellectual or developmental disability. The Fund encourages quality and innovation in the disability services sector, by providing grants to researchers to undertake research that will advance the health, well-being and life experiences of people with a disability. The Fund also provides grants to nonprofit organisations to expand their activities to include people with a disability, or to deliver innovative projects that enhance social inclusion for people with a disability.

Authors' contributions:

Protocol concept and design: LM, NS, JM, GD

Search strategy development: LM, NS, JM, GD

Manuscript writing: LM, NS

Review and editing of protocol and manuscript: LS, NS, JM, GD

All authors read and approved the final manuscript.

Acknowledgements: The authors would like to acknowledge Alison Forrest and Liz Norsa who assisted with conducting initial and preliminary searches, and early categorisation of literature topics and populations. 


\section{References}

1. Arnett JJ. Emerging adulthood: The winding road from the late teens through the twenties: Oxford University Press; 2015.

2. Buock EC, Joshi GS. Transition and Students With Mild Intellectual Disability: Findings From the National Longitudinal Transition Study-2. Career Development and Transition for Exceptional Individuals. 2016;39(3):154-63.

3. Pallisera M, Fullana J, Puyalto C, Vila M. Changes and challenges in the transition to adulthood: views and experiences of young people with learning disabilities and their families. European Journal of Special Needs Education,. 2016;31(3):391-406.

4. Health Education England. Learning Disability Transition Pathway Competency Framework United Kingdom: National Institute for Health and Care Excellence (NICE); 2016 [Available from: https://www.nice.org.uk/sharedlearning/learning-disability-transitionpathway-competency-framework.

5. NSW Government. Transition planning NSW Government Website - Education: NSW Government; 2016 [updated 15.07.2021. Available from: https://education.nsw.gov.au/teaching-and-learning/disability-learning-and-support/leaving-school/transitionplanning.

6. U.S. Department of Education - Office for Civil Rights. Transition of Students With Disabilities To Postsecondary Education: A Guide for High School Educator Washington, DC, United States of America2011 [Available from: https://www2.ed.gov/about/offices/list/ocr/transitionguide.html.

7. Meadows CA, Fraser J, Camus S, Henderson K. A system-wide innovation in transition services: Transforming the home care liaison role. Home Healthcare Now. 2014;32(2).

8. Dyke P, Bourke J, Llewellyn G, Leonard $\mathrm{H}$. The experiences of mothers of young adults with an intellectual disability transitioning from secondary school to adult life. Journal of Intellectual and Developmental Disability. 2013;38(2):149-62.

9. Ashburner JK, Bobir NI, van Dooren K. Evaluation of an Innovative Interest-Based Post-School Transition Programme for Young People with Autism Spectrum Disorder. International Journal of Disability, Development and Education,. 2018;65(3):262-85.

10. Gauthier-Boudreault C, Gallagher F, Couture M. Specific needs of families of young adults with profound intellectual disability during and after transition to adulthood: What are we missing? Research in Developmental Disabilities. 2017;66:16-26.

11. Leonard H, Foley K, Pikora T, Bourke J, Wong K, McPherson L, et al. Transition to adulthood for young people with intellectual disability: the experiences of their families. European Child Adolescent Psychiatry. 2016;25:1369-81.

12. Brooker K, van Dooren K, Tseng C-H, McPherson L, Lennox N, Ware R. Out of sight, out of mind? The inclusion and identification of people with intellectual disability in public health research. Perspectives in public health. 2015;135(4):204-11.

13. McDonald KE, Conroy NE, Olick RS, Panel PEE. Is it worth it? Benefits in research with adults with intellectual disability. Intellectual and developmental disabilities. 2016;54(6):440-53.

14. McDonald KE, Kidney CA, Patka M. 'You need to let your voice be heard': research participants' views on research. Journal of Intellectual Disability Research. 2013;57(3):216-25.

15. McDonald KE, Kidney CA. What Is Right? Ethics in Intellectual Disabilities Research. Journal of Policy and Practice in Intellectual Disabilities. 2012;9(7):27-39.

16. Schwartz AE, Kramer JM, Cohn ES, McDonald KE. "That felt like real engagement": Fostering and maintaining inclusive research collaborations with individuals with intellectual disability. Qualitative health research. 2020;30(2):236-49.

17. Bigby C, Frawley P, Ramcharan P. Conceptualizing inclusive research with people with intellectual disability. Journal of Applied Research in Intellectual Disabilities. 2014;27(1):3-12.

18. Australian Parliament. The National Disability Insurance Scheme: a quick guide: Australian Parliament; 2017 [Available from: https://www.aph.gov.au/About_Parliament/Parliamentary_Departments/Parliamentary_Library/pubs/rp/rp1617/Quick_Guides/NDIS.

19. Ramcharan P. Understanding the NDIS: a history of disability welfare from 'deserving poor' to consumers in control. The Conversation. 2016 July 6, 2016.

20. Poirier S-Ė, St-Pierre M-C, Julien-Gauthier F, Flamand V, Martin-Roy S, Desmarais C. Inter-Agency Collaboration in the Transition from School to Adulthood of Students with Disabilities: A Narrative Review of the Literature. International Journal of Disability, Development and Education. 2020:1-8.

21. Moher D, Shamseer L, Clarke M, Ghersi D, Liberati A, Petticrew M, et al. Preferred Reporting Items for Systematic Review and MetaAnalysis Protocols (PRISMA-P) 2015 statement. BMC Systematic reviews. 2015;4(1):1(1).

22. Arksey H, O'Malley L. Scoping Studies: Towards a Methodological Framework. International Journal of Social Research Methodology. 2005;8(1):19-32. 
23. Peters MDJ, Godfrey C, Mclnerney P, Munn Z, Tricco A, Khalil H. Chapter 11: Scoping Reviews (2020 version). In: Aromataris E, Munn Z, editors. JBI Manual for Evidence Synthesis, JBI2020. p. https://synthesismanual.jbi.global. https://doi.org/10.46658/JBIMES-2012.

24. Levac D, Colquhoun H, O'Brien KK. Scoping studies: Advancing the methodology. Implementation Science. $2010 ; 5(1): 69$.

25. Arnett JJ. Presidential address: The emergence of emerging adulthood: A personal history. Emerging Adulthood. 2014;2(3):155-62.

26. Erickson G, Macmillan R. Disability and the transition to adulthood: A life course contingency perspective. Longitudinal and Life Course Studies. 2018;9(2):188-211.

27. Diament M. Obama Signs Bill Replacing 'Mental Retardation' With 'Intellectual Disability'2010 August 11, 2021. Available from: https://www.disabilityscoop.com/2010/10/05/obama-signs-rosas-law/10547/.

28. Social Security Administration - Federal Register. Change in Terminology: "Mental Retardation" to "Intellectual Disability". 78 FR 46499. 2013:46499-502 (4 pages).

29. United Nations. Convention on the rights of persons with disabilities. A/61/611. 2006;http://www.un.org/esa/socdev/enable/rights/convtexte.htm\#optprotocol.

30. Shier H. Pathways to participation: Openings, opportunities and obligations. Children \& society. 2001;15(2):107-17.

31. United Nations. Convention on the rights of the Child. 1989;https://www.ohchr.org/EN/Professionallnterest/Pages/CRC.aspx.

32. Straus SE, Tetroe J, Graham I. Defining knowledge translation. Canadian Medical Association Journal. 2009;181(3-4):165-8.

33. Dew A, Boydell KM. Knowledge translation: Bridging the disability research-to-practice gap. Research and Practice in Intellectual and Developmental Disabilities. 2017;4(2):142-57.

\section{Supplementary}

Additional file 6 is not available with this version

\section{Supplementary Files}

This is a list of supplementary files associated with this preprint. Click to download.

- Additionalfile1PRISMAP2015fillablechecklist.docx

- Additionalfile2scopingreviewframework.docx

- Additionalfile3PrelimSearchCINAHL.pdf

- Additionalfile4PRISMAScRFillableChecklist1a.docx

- Additionalfile5PRISMA2009FlowDiagram.doc 